\title{
The Anisotropic Two-Point Correlation Functions of the Nonlinear Traceless Tidal Field in the Principal-Axis Frame
}

\author{
Jounghun Lee ${ }^{1}$, Oliver Hahn ${ }^{2}$, And Cristiano Porciani ${ }^{2,3}$ \\ jounghun@astro.snu.ac.kr
}

\begin{abstract}
Galaxies on the largest scales of the Universe are observed to be embedded in the filamentary cosmic web which is shaped by the nonlinear tidal field. As an efficient tool to quantitatively describe the statistics of this cosmic web, we present the anisotropic two-point correlation functions of the nonlinear traceless tidal field in the principal-axis frame, which are measured using numerical data from an N-body simulation. We show that both of the nonlinear density and traceless tidal fields are more strongly correlated along the directions perpendicular to the eigenvectors associated with the largest eigenvalues of the local tidal field. The correlation length scale of the traceless tidal field is found to be $\sim 20 h^{-1} \mathrm{Mpc}$, which is much larger than that of the density field $\sim 5 h^{-1} \mathrm{Mpc}$. We also provide analytic fitting formulae for the anisotropic correlation functions of the traceless tidal field, which turn out to be in excellent agreement with the numerical results. We expect that our numerical results and analytic formula are useful to disentangle cosmological information from the filamentary network of the large-scale structures.
\end{abstract}

Subject headings: cosmology:theory — large-scale structure of universe

\section{INTRODUCTION}

On the largest scales, the spatial distribution of galaxies exhibits the pattern of a filamentary network which is commonly referred to as the "cosmic web". N-body simulations have long predicted the web-like pattern in the clustering of matter and galaxies (e.g.,

\footnotetext{
${ }^{1}$ Department of Physics and Astronomy, FPRD, Seoul National University, Seoul 151-742, Korea

${ }^{2}$ Institute for Astronomy, ETH Zurich, CH-8093 Zürich, Switzerland

${ }^{3}$ Argelander Institut für Astronomie, Auf dem Hügel 71, D-53121 Bonn, Germany
} 
Shandarin \& Zel'Dovich 1986), recent large galaxy surveys have confirmed its presence (e.g., Colless et al. 2001), and theoretical endeavors have been able to explain the naturalness of its occurrence in the context of the cold dark matter (CDM) paradigm (Bond \& Myers 1996; Bond et al. 1996). It is now well accepted that the cosmic web is a real, natural, and common phenomenon.

Nonetheless, the most crucial part has been missing for the comprehension of the cosmic web - an optimal statistical tool to quantify it. The standard N-point statistics of course can be used in principle to quantify it, but they cannot capture the essence of the cosmic web that it is a nonlinear manifestation of the primordial tidal interactions. It is because in N-point statistics what is emphasized is the role of gravity in establishing the high-order correlations of the density field. The cosmic web is a nonlinear manifestation of the spatial coherence of the tidal field (Bond et al. 1996). Thus, to quantify the cosmic web and capture its essence, it is necessary to have a statistical tool by which the role and effect of the tidal field and its spatial correlation are highlighted.

Patchy efforts have so far been made to account for the effect of the tidal field on structure formation. For instance, the failure of the Press-Schecther mass function (Press \& Schechter 1974) has been attributed to the deviation of the gravitational process from spherical dynamics caused by the effect of the tidal forces (Monaco 1995; Audit et al. 1997; Lee \& Shandarin 1998; Sheth et al. 2001). The galaxy angular momentum has been ascribed to the initial tidal interactions with the surrounding matter distribution (Doroshkevich 1970; White 1984; Catelan \& Theuns 1996; Lee \& Pen 2000; Porciani et al. 2002a, b). The intrinsic galaxy alignments are also found to be explained due to the effect of the nonlinear tidal interactions between the galaxies. (Catelan et al. 2001; Crittenden et al. 2001; Lee et al. 2005; Lee \& Pen 2007, 2008). The alignment and ellipticity correlation functions have been developed to quantify the preferential orientations of galaxies relative to the surrounding large-scale structure (Faltenbacher et al. 2009). The cosmic web, however, requires not a patchy but a unified way in which the multiple aspects of the nonlinear tidal field can be quantified.

The difficulty in quantifying the effect of the tidal field lies in the fact that the correlations of the tidal field are highly anisotropic even in the linear regime. Lee \& Pen (2001) and Catelan \& Porciani (2001) have for the first time derived analytically the anisotropic twopoint correlations of the linear tidal field. But, their results were all expressed in the fixed Cartesian coordinate frame. To fully appreciate the anisotropic nature of the correlations of the tidal field, it is desirable to find the correlations of the tidal field in the principal axis frame, since the filamentary cosmic web is generated by the anisotropic collapse of matter along the principal axes of the tidal field (Bond et al. 1996). Unfortunately, it is extremely difficult to derive analytically the correlations of even the linear tidal field in the principal 
axis frame which rotates from point to point.

The goal of this Paper is to measure numerically the anisotropic two-point correlations of the nonlinear tidal field in the principal-axis frame and to provide an analytic fitting formula as an efficient statistical tool for the description of the cosmic web. This paper is organized as follows. In $\S 2$, we provide a brief description of the numerical data. In $\S 3$, we present the numerical results and analytic fitting formula as well. In $\S 4$, we summarize the results and discuss the implications.

\section{DATA}

We use the N-body results derived by Hahn et al. (2007) using the tree-PM code GADGET2 (Springel et al. 2005). The simulations followed the evolution of $512^{3}$ particles starting from redshift $z=52.4$ to $z=0$ in a periodic box of linear size $180 h^{-1}$ Mpc. A flat $\Lambda$ CDM cosmology is assumed with key parameters given as $\Omega_{m}=0.25$ (matter density), $\Omega_{\Lambda}=0.75$ (vacuum energy density ), $\Omega_{b}=0.045$ (baryon density), $\sigma_{8}=0.9$ (power spectrum amplitude), $H_{0}=0.73$ (Hubble constant) and $n_{s}=1$ (spectral index).

Hahn et al. (2007) constructed the overdensity field $\delta$ (dimensionless density contrast) on $1024^{3}$ grids with the help of the Cloud-In-Cell interpolation method and smoothed it by a Gaussian kernel with a filtering radius $R_{\mathrm{s}}$. The peculiar gravitational potential field $\phi$ was also derived from the overdensity field by solving the Poisson's equation $\nabla^{2} \phi \propto \delta$. In simulations, the overdensity field is converted to the Fourier space with the help of a fast Fourier transform (FFT). The potential field is obtained as $\phi \propto k^{-2} \delta$ where $k$ represents the magnitude of the wave vector in the Fourier space. For a detailed description of the N-body simulations and the derivation of the density and potential fields, see Hahn et al. (2007). For the smoothing of the density (and potential) fields, we use the small smoothing scale $R_{s}=0.5 h^{-1} \mathrm{Mpc}$ to minimize the effect of the smoothing on the determination of the correlations of the tidal fields.

The tidal tensor $\left(T_{i j}\right)$ is defined as the Hessian of the peculiar gravitational potential $T_{i j} \equiv \partial_{i} \partial_{j} \phi$. In Fourier space, it is written as $T_{i j}=k_{i} k_{j} \phi$. Using the FFT again, we calculate the tidal tensor at each of the randomly selected 250000 grid points. The sum of the diagonal components of $\left(T_{i j}\right)$ (i.e., the trace of the tidal field) equals the dimensionless density contrast, $\delta$. To sort out the true tidal effect from the gravitational effect, we calculate the traceless tidal field $\left(\tilde{T}_{i j}\right)$ as $\tilde{T}_{i j} \equiv T_{i j}-(\delta / 3) I_{i j}$ where $\left(I_{i j}\right)$ denotes the identity tensor. Diagonalizing $\tilde{T}_{i j}$ at each of the 250000 sampling points, we find its three eigenvalues, $\left\{\lambda_{1}, \lambda_{2}, \lambda_{3}\right\}$ (in a decreasing order, i.e., $\lambda_{1}>\lambda_{2}>\lambda_{3}$ ), and the corresponding normalized 
eigenvectors with unit magnitude, $\left\{\hat{\mathbf{e}}_{1}, \hat{\mathbf{e}}_{2}, \hat{\mathbf{e}}_{3}\right\}$. Regardless of whether a given volume element is expanding or contracting (negative or positive $\delta$ ) the traceless tidal tensor $\tilde{T}_{i j}$ gives the deformation of that volume element (relative to spherical expansion or contraction). By construction, we have $\lambda_{1}>0$ and $\lambda_{3}<0$. The second largest eigenvalue $\lambda_{2}$ can take on either sign. The maximal (relative) compression of a given volume element by gravity occurs along the direction parallel to $\hat{\mathbf{e}}_{1}$ associated with $\lambda_{1}$. Figure 1 plots the probability density distributions of $\lambda_{1}, \lambda_{2}$ and $\lambda_{3}$ as solid, dashed and dotted lines, respectively. The probability density distribution of $\lambda_{1}$ and $\lambda_{3}$ is positively and negatively skewed, respectively while that of $\lambda_{2}$ is almost symmetric about zero. Note that the two distributions, $p\left(\lambda_{1}\right)$ and $p\left(\lambda_{3}\right)$, are also almost symmetric to each other around zero: $p\left(\lambda_{1}\right) \sim p\left(-\lambda_{3}\right)$. The distribution $p\left(\lambda_{2}\right)$ is much narrower than $p\left(\lambda_{1}\right)$.

In the following section, we measure the two-point correlation functions of $\lambda_{1}, \lambda_{2}, \lambda_{3}$ and $\delta$ in the principal-axis frame of the traceless tidal field and study their characteristic behaviors.

\section{NUMERICAL ANALYSIS AND FITTING FORMULA}

For each pair of points located at $\mathbf{x}$ and $\mathbf{x}+\mathbf{r}$, we express the separation vector $\mathbf{r}$ in the frame of the principal axes of $\tilde{T}_{i j}(\mathbf{x})$ spanned by $\left\{\hat{\mathbf{e}}_{1}, \hat{\mathbf{e}}_{2}, \hat{\mathbf{e}}_{3}\right\}$ with orienting the $z$-axis in the direction of $\hat{\mathbf{e}}_{1}$ (the first principal axis of the local tidal field). Let $(r, \theta, \phi)$ represent the spherical polar coordinate of $\mathbf{r}$ in the principal-axis frame. For every grid pair, we calculate the product, $\lambda_{i}(\mathbf{x}) \lambda_{i}(\mathbf{x}+\mathbf{r})$ (for $\left.i=1,2,3\right)$. Then, we take the average of the product over the points whose separation vectors belong to the differential bin, $[\mathbf{r}, \mathbf{r}+d \mathbf{r}]$, to determine the correlation of $\lambda_{i}$ as a function of $(r, \theta, \phi)$ as

$$
\xi_{\lambda i}(r, \theta, \phi)=\left\langle\lambda_{i}(\mathbf{x}) \cdot \lambda_{i}(\mathbf{r}+\mathbf{x})\right\rangle
$$

Figure 2 shows the isotropic two-point correlations $\xi_{\lambda i}(r)$ of the three eigenvalues of the traceless tidal field as a function of $r$ (solid, dashed and dotted line for $\lambda_{1}, \lambda_{2}$ and $\lambda_{3}$, respectively) at $z=0$. This isotropic correlation function $\xi_{\lambda i}(r)$ is obtained by taking the average $\lambda_{i}(\mathbf{x}) \lambda_{i}(\mathbf{x}+\mathbf{r})$ over the points whose separation distances belong to the differential distance bin, $[r, r+d r]$. The tendency of $\xi_{\lambda i}(r)$ being flat for $r<2 h^{-1} \mathrm{Mpc}$ is due to the effect of the smoothing of the tidal field. The isotropic two-point correlation of $\delta$ is also plotted for comparison (thin solid line). As it can be seen, the correlations of $\lambda_{1}$ and $\lambda_{3}$ decrease with $r$

much more slowly than that of $\delta$. In other words, the largest and smallest eigenvalues of the traceless tidal field are coherent over larger scales than the density field $\delta$. Let the correlation length scale $l_{c}$ correspond to the distance at which the correlation strength becomes an order 
of magnitude lower than its value at $r=0$. We find that $l_{c} \approx 20 h^{-1} \mathrm{Mpc}$ for $\lambda_{1}$ and $\lambda_{3}$, while $\delta$ and $\lambda_{2}$ has $l_{c} \approx 3 h^{-1} \mathrm{Mpc}$. The smallest eigenvalue $\lambda_{3}$ has the highest amplitude at all distances, while the second largest eigenvalue $\lambda_{2}$ has the lowest amplitude at $r \leq 10 h^{-1} \mathrm{Mpc}$. The correlation amplitude of $\delta$ is comparable to that of $\lambda_{1}$ at small distance $r \leq 2 h^{-1} \mathrm{Mpc}$ but decreases much more rapidly with $r$ than $\lambda_{1}$, reaching the lowest value at $r \geq 10 h^{-1} \mathrm{Mpc}$.

For the rescaled isotropic correlation $\xi_{\lambda i}(r) / \xi_{\lambda i}(0)$, we find the following analytic formula:

$$
\begin{aligned}
& \frac{\xi(r)}{\xi(0)}=\left(\frac{r}{r_{0}}+1\right)^{n} r^{n-3}, \text { for } \lambda_{1}, \lambda_{3}, \\
& \frac{\xi(r)}{\xi(0)}=\left(\frac{r}{r_{0}}+1\right)^{n} r^{1 / 2-n}, \text { for } \lambda_{2}
\end{aligned}
$$

where $r_{0}$ and $n$ are two fitting parameters. By comparing the numerical results with the above analytic formula, we empirically determine the best-fit values of $r_{0}$ and $n$, which are listed in Table 1. Figure 3 shows the rescaled isotropic correlations of $\lambda_{1}$ (left), $\lambda_{2}$ (middle) and $\lambda_{3}$ (right) at $z=0$. In each panel the dots and the solid line represent the numerical results and the analytic model with best-fit parameters, respectively. The error at each bin is calculated as one standard deviation in the measurement of the mean value. As it can be seen, the analytic models (eqs. 2. 3]) fit the numerical results very well for $r \geq 2 h^{-1} \mathrm{Mpc}$. On smaller distances $r<2 h^{-1} \mathrm{Mpc}$, the numerical results become flatter than the analytic curve due to the smoothing effect.

Figure 4 shows the anisotropic two-point correlations of $\lambda_{1}$ (left), $\lambda_{2}$ (middle) and $\lambda_{3}$ (right) as a function of $r$ and their variation with $\theta$ at $z=0$. These anisotropic correlations $\xi_{\lambda i}(r, \theta)$ are obtained by taking the average of $\lambda_{i}(\mathbf{x}) \lambda_{i}(\mathbf{x}+\mathbf{r})$ over the points whose separation distances belong to the differential distance bin $[r, r+d r]$ and at which the angles between the separation vectors and the first principal axes are in the differential range of $[\theta, \theta+d \theta]$. In each panel the thick solid, dashed, dotted, longdashed, dot-dashed, and dot-longdashed line correspond to the range $0^{\circ} \leq \theta<15^{\circ}, 15^{\circ} \leq \theta<30^{\circ}, 30^{\circ} \leq \theta<45^{\circ}, 45^{\circ} \leq \theta<60^{\circ}$, $60^{\circ} \leq \theta<75^{\circ}$ and $75^{\circ} \leq \theta \leq 90^{\circ}$, respectively. The fluctuations of $\xi_{\lambda_{i}}(r, \theta)$ in the small- $r$ section $\left(r \leq 2 h^{-1} \mathrm{Mpc}\right)$ are due to the numerical noise. The anisotropic two-point correlation of $\delta$ is also plotted for comparison as thin lines in each panel. As it can be seen, both correlations of $\lambda$ and $\delta$ increase with $\theta$ in the principal-axis frame at all distances. That is, the correlations become stronger toward the directions normal to the first principal axes of the tidal field. This behavior is consistent with the scenario that matter compress maximally along the first principal axes of the tidal field and thus is more likely to lie in the plane normal to the first principal axis of the tidal field. The variation of $\lambda_{2}$ with $\theta$ is stronger than that of $\lambda_{1}$ and $\lambda_{3}$. Note also that the degree of variation of $\xi_{\lambda_{i}}(r, \theta)$ with $\theta$ decreases with $r$. In other words, the correlations tend to become less anisotropic at large distances $\left(r \geq 10 h^{-1} \mathrm{Mpc}\right)$. 
We also obtain $\xi_{\lambda_{i}}(\theta)$ by taking the average of $\lambda_{i}(\mathbf{x}) \lambda_{i}(\mathbf{x}+\mathbf{r})$ over the points at which the angles between the first principal axis and the separation vectors belong to the differential angle bin, $[\theta, \theta+d \theta]$. The following analytic formulae are found to fit the numerical results well:

$$
\begin{aligned}
& \frac{\xi(\theta)}{\xi(0)}=\frac{1}{1-A \sin ^{4} \theta}, \text { for } \lambda_{1}, \lambda_{3}, \\
& \frac{\xi(\theta)}{\xi(0)}=\frac{1}{1-A \sin ^{2} \theta}, \text { for } \lambda_{2}
\end{aligned}
$$

where $A$ is an adjustable parameter whose best-fit value is determined via the $\chi^{2}$ statistics (see Table 1). Figure 4 shows the anisotropic correlation function of $\lambda_{1}$ (right), $\lambda_{2}$ (middle) and $\lambda_{3}$ (left) as a function of $\theta$ in the principal axis frame of the local tidal field. In each panel the dots represent the numerical results while the solid line is the analytic fitting formula. As it can be seen, the analytic models fit the numerical results very well for each case. To improve readibility we do not show errorbars which are negligibly small anyway. These results show clearly how anisotropic the correlations of the eigenvalues of the traceless tidal field are with respect to the first principal axis. The difference between the correlations at $\theta=0$ and $\theta=90^{\circ}$ reaches approximately $60 \%$ for $\lambda_{1}$ and $\lambda_{3}$, while it increases up to $70 \%$ for $\lambda_{2}$.

\section{SUMMARY AND DISCUSSION}

The web-like pattern in the large-scale matter distribution is an imprint produced by the effect of the nonlinear tidal field in the late universe. While the trace part of the tidal field is responsible for the collapse of matter, its traceless part deforms the cosmic volume elements, resisting the overall spherical expansion or contraction. In consequence the traceless tidal field induces anisotropy in the large-scale correlations of matter distribution. An efficient statistical tool has long been looked for to describe quantitatively this cosmic web and to retrieve cosmological information encoded in the cosmic web.

We have determined numerically the two-point correlations of the three eigenvalues of the nonlinear traceless tidal field defined as the Hessian of the gravitational potential in the frame of the principal axes of the tidal field. The numerical findings indicate that the correlation functions of the traceless tidal field and the density field are all anisotropic relative to the principal axes. Their correlations having much larger correlation length scales than that of the density field. increase along the directions normal to the first principal axes of the tidal field. The analytic fitting formula for the correlation functions of the three eigenvalues of the tidal field are determined and found to fit the numerical results well. 
Our numerical and analytical results are thus able to provide a physical description of the large-scale filamentary structure.

It will be interesting to know how the anisotropic two-point correlations of thetraceless tidal field depend on the background cosmology. Our future work is in this direction.

J.L. acknowledges the financial support from the Korea Science and Engineering Foundation (KOSEF) grant funded by the Korean Government (MOST, NO. R01-2007-000-102460). O.H. acknowledges support from the Swiss National Science Foundation. All simulations were performed on the Gonzales cluster at ETH Zurich, Switzerland. 


\section{REFERENCES}

Aragón-Calvo, M. A., Jones, B. J. T., van de Weygaert, R., \& van der Hulst, J. M. 2007, A\&A, 474, 315

Audit, E., Teyssier, R., \& Alimi, J.-M. 1997, A\&A, 3

Barrow, J. D., Bhavsar, S. P., \& Sonoda, D. H. 1985, MNRAS, 216, 17

Bond, J. R., Kofman, L., \& Pogosyan, D. 1996, Nature, 380, 603

Bond, J. R., \& Myers, S. T. 1996, ApJS, 103, 1

Catelan, P., \& Theuns, T. 1996, MNRAS, 282, 436

Catelan, P., Kamionkowski, M., \& Blandford, R. D. 2001, MNRAS, 320, L7

Catelan, P., \& Porciani, C. 2001, MNRAS, 323, 713

Colless, M., et al. 2001, MNRAS, 328, 1039

Colberg, J. M. 2007, MNRAS, 375, 337

Crittenden, R. G., Natarajan, P., Pen, U.-L., \& Theuns, T. 2001, ApJ, 559, 552

Doroshkevich, A. G. 1970, Astrofizika, 6, 581

Faltenbacher, A., Li, C., White, S. D. M., Jing, Y.-P., Shu-DeMao, \& Wang, J. 2009, Research in Astronomy and Astrophysics, 9, 41

Hahn, O., Porciani, C., Carollo, C. M., \& Dekel, A. 2007, MNRAS, 375, 489

Hahn, O., Porciani, C., Dekel, A., \& Carollo, C. M. 2008, arXiv:0803.4211

Jing, Y. P. 2002, MNRAS, 335, L89

Lee, J., \& Pen, U.-L. 2000, ApJ, 532, L5

Lee, J., \& Shandarin, S. F. 1998, ApJ, 500, 14

Lee, J., Kang, X., \& Jing, Y. P. 2005, ApJ, 629, L5

Lee, J. 2006, ApJ, 644, L5

Lee, J., \& Park, D. 2006, ApJ, 652, 1

Lee, J., \& Pen, U.-L. 2001, ApJ, 555, 106 
Lee, J., \& Pen, U.-L. 2007, ApJ, 670, L1

Lee, J., Springel, V., Pen, U.-L., \& Lemson, G. 2008, MNRAS, 389, 1266

Lee, J., \& Pen, U.-L. 2008, ApJ, 681, 798

Navarro, J. F., Abadi, M. G., \& Steinmetz, M. 2004, ApJ, 613, L41

Monaco, P. 1995, ApJ, 447, 23

Nichol, R. C., et al. 2006, MNRAS, 368, 1507

Roberts, S., Davies, J., Sabatini, S., Auld, R., \& Smith, R. 2007, MNRAS, 379, 1053

Porciani, C., Dekel, A., \& Hoffman, Y. 2002, MNRAS, 332, 325

Porciani, C., Dekel, A., \& Hoffman, Y. 2002, MNRAS, 332, 339

Press, W. H., \& Schechter, P. 1974, ApJ, 187, 425

Shandarin, S. F., \& Zel'Dovich, I. B. 1986, European Southern Observatory Astrophysics Symposia, 23, 45

Sheth, R. K., Mo, H. J., \& Tormen, G. 2001, MNRAS, 323, 1

Springel, V., 2005, MNRAS, 364, 1105

Springel, V., et al. 2005, Nature, 435, 629

Tanaka, M., Hoshi, T., Kodama, T., \& Kashikawa, N. 2007, MNRAS, 379, 1546

Trujillo, I., Carretero, C., \& Patiri, S. G. 2006, ApJ, 640, L111

White, S. D. M. 1984, ApJ, 286, 38

This preprint was prepared with the AAS LATEX macros v5.2. 


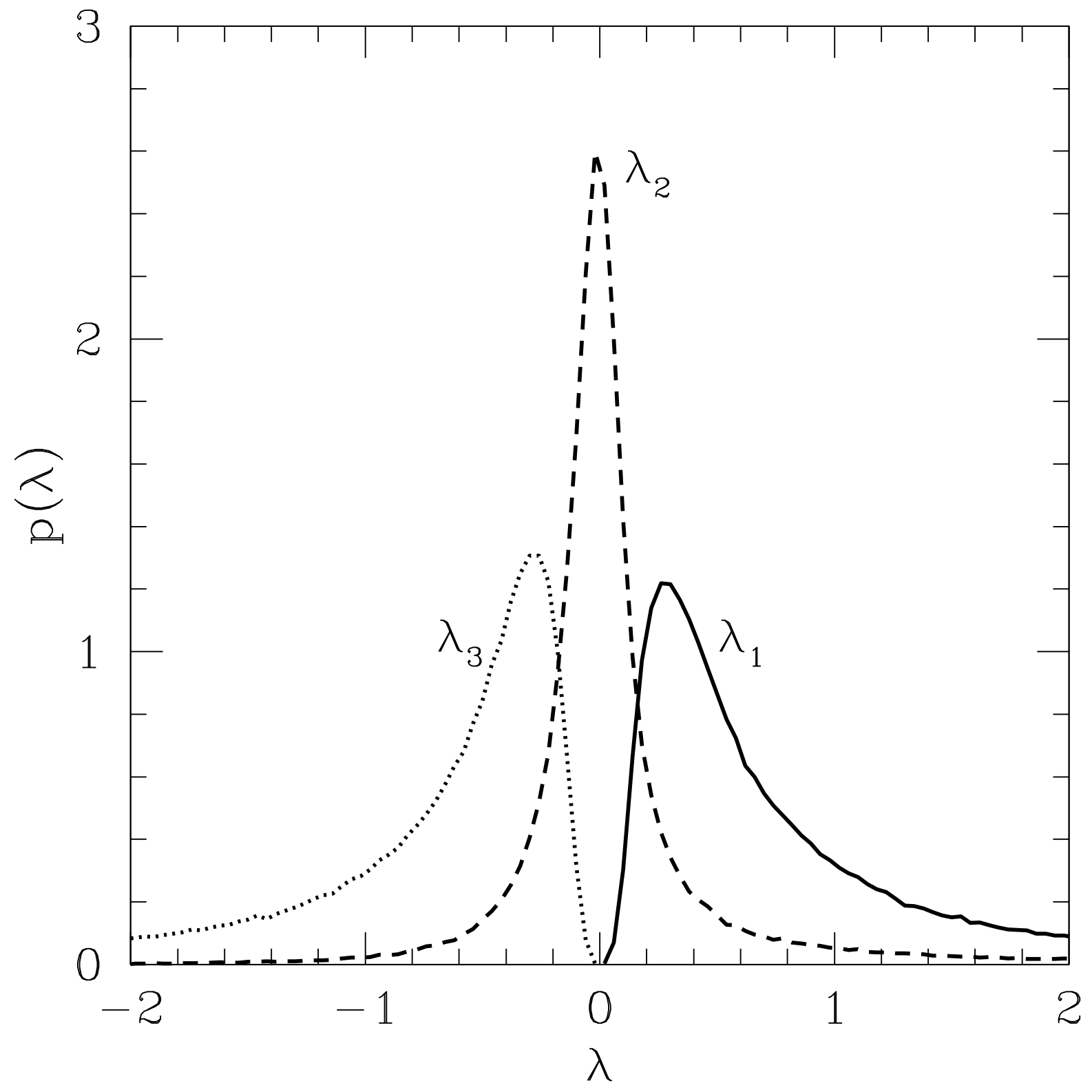

Fig. 1.- Probability density distributions of the three eigenvalues, $\lambda_{1}, \lambda_{2}$ and $\lambda_{3}$ (solid, dotted and dashed line, respectively) of the traceless tidal field smoothed on the scale of $0.5 h^{-1} \mathrm{Mpc}$. 


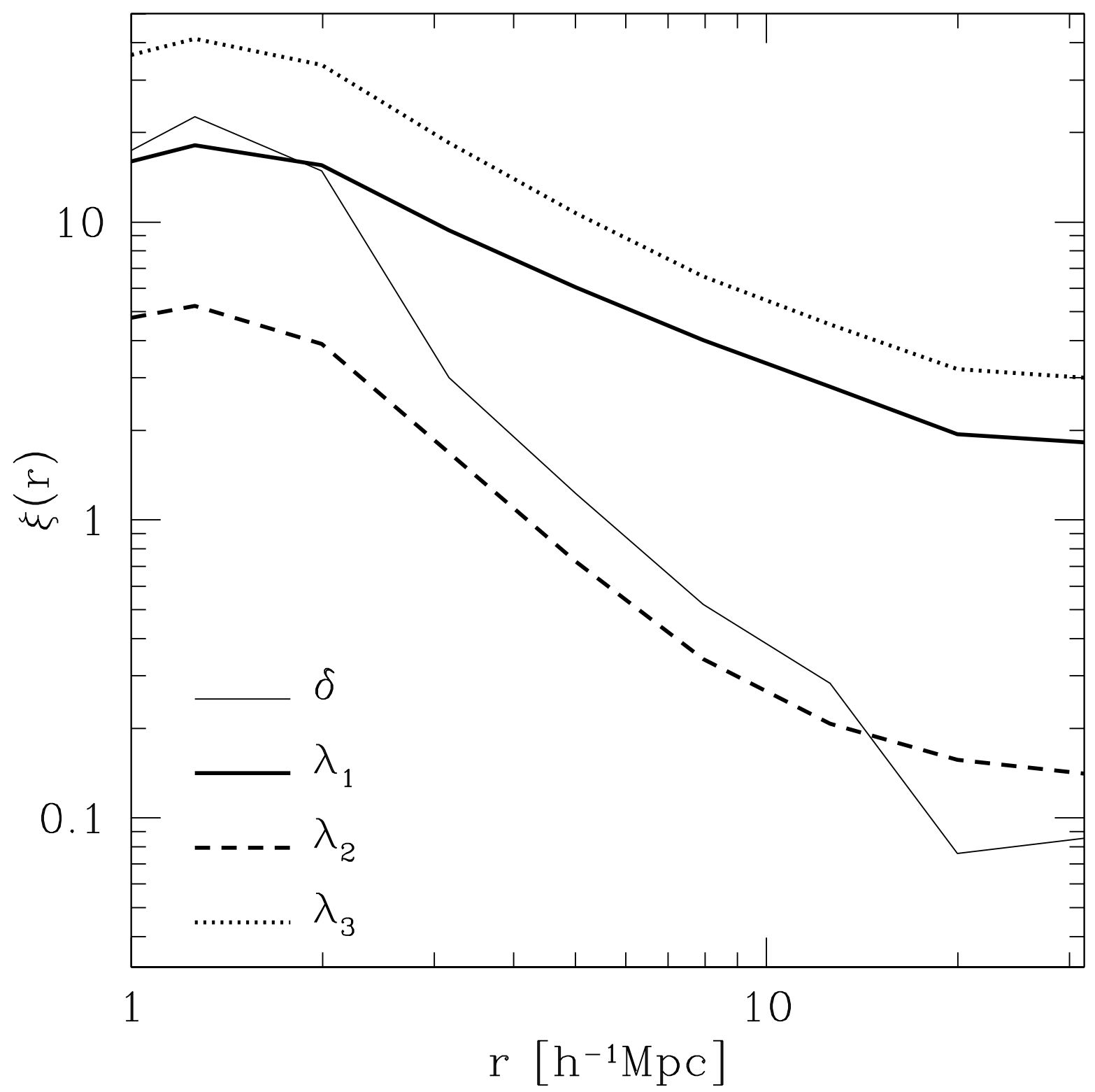

Fig. 2.- Two-point isotropic correlation functions of the three eigenvalues of the traceless tidal field, $\lambda_{1}, \lambda_{2}$ and $\lambda_{3}$ (solid, dashed and dotted line, respectively) smoothed on the scale of $0.5 h^{-1} \mathrm{Mpc}$. The two-point isotropic correlations of $\delta$ is also plotted (thin solid line) for comparison. 


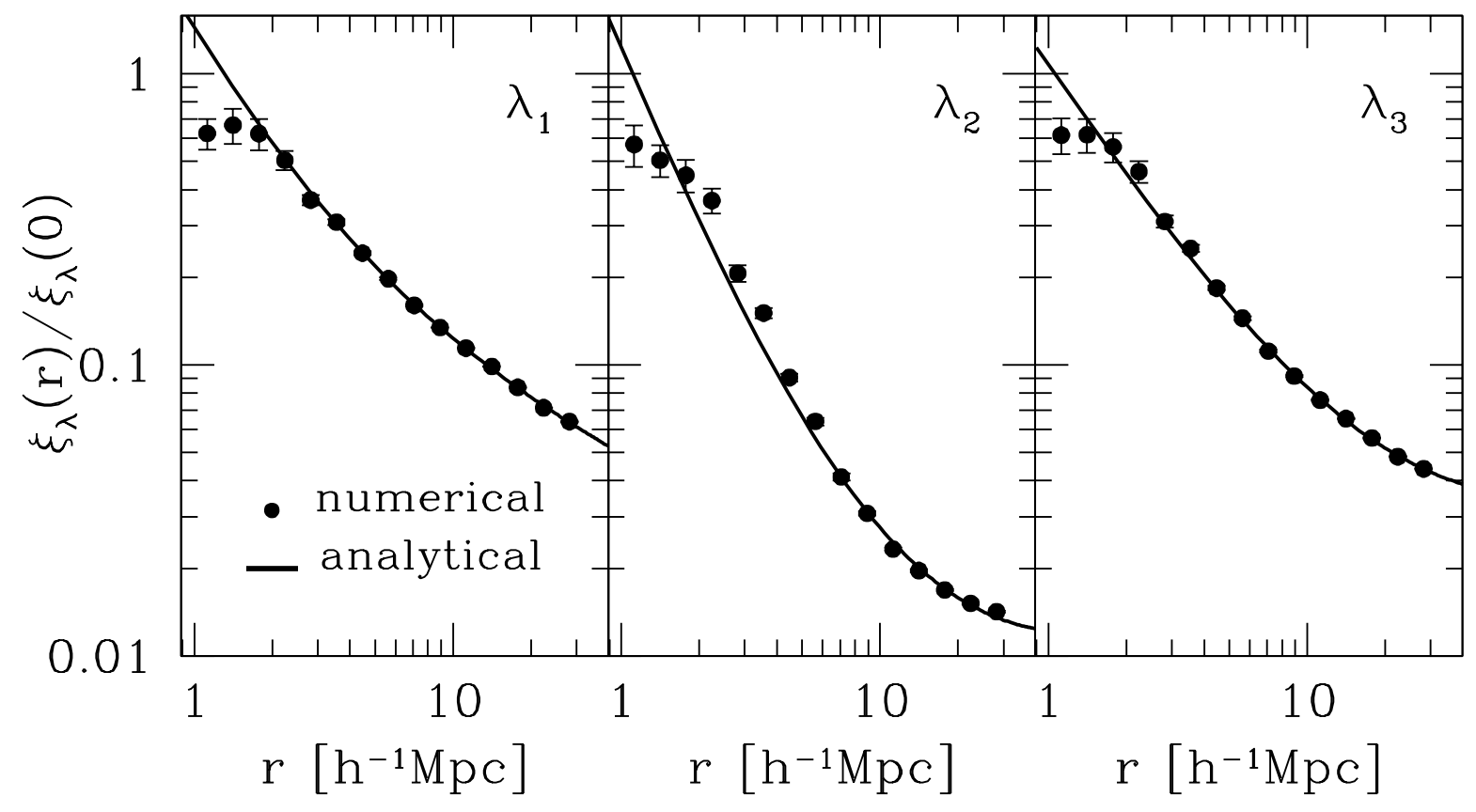

Fig. 3.- Rescaled isotropic correlation functions of $\lambda_{1}$ (right), $\lambda_{2}$ (middle) and $\lambda_{3}$ (left) in the principal-axis frame of the local tidal field. In each panel the dots represent the numerical results while the solid line is the analytic fitting formula. The errors represent one standard deviation in the measurement of the mean values. 


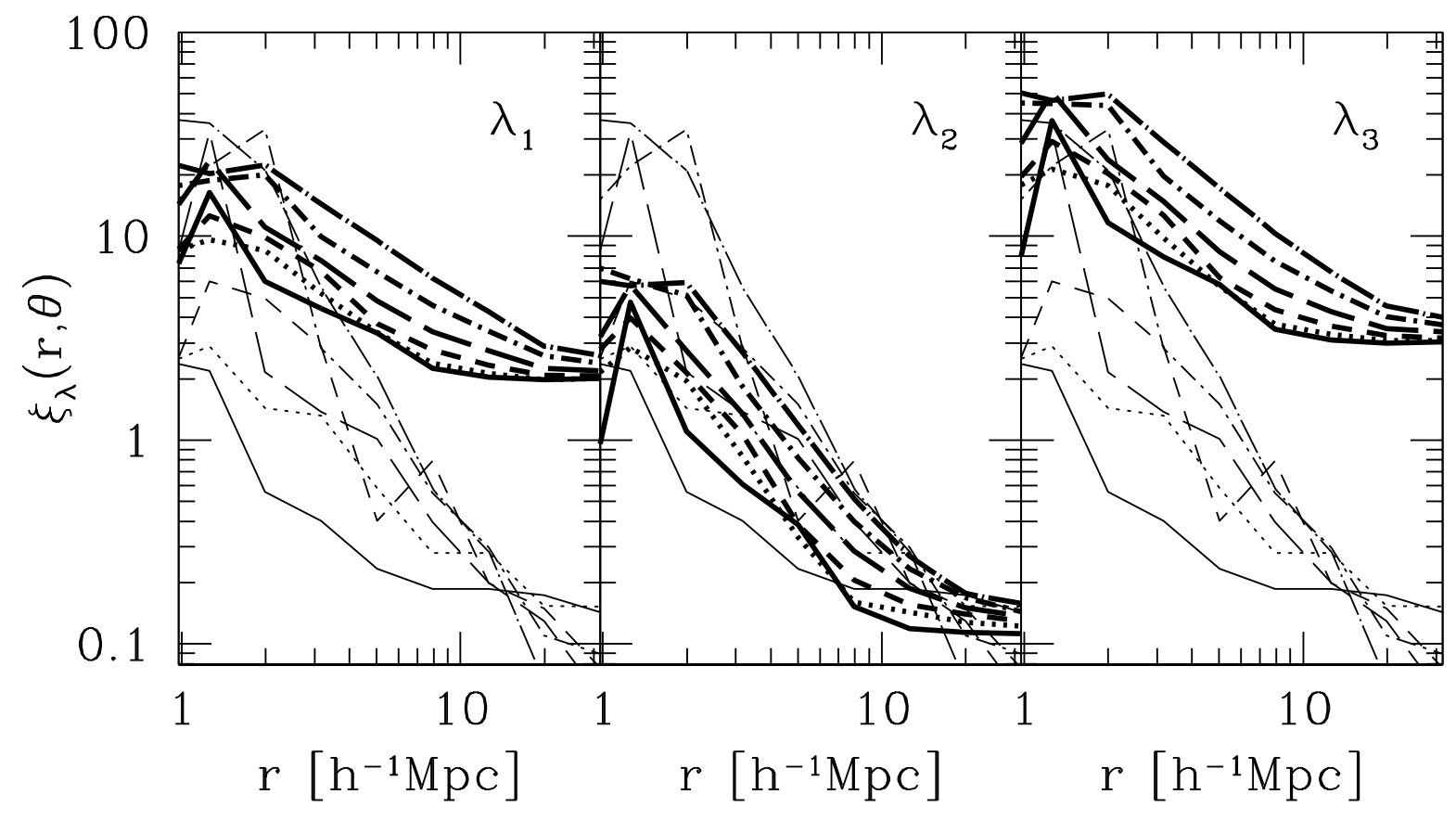

Fig. 4.- Anisotropic correlation functions of $\lambda_{1}$ (right), $\lambda_{2}$ (middle) and $\lambda_{3}$ (left) as a function of $r$ and their variations with polar angle $\theta$ in the principal-axis frame of the local tidal field (thick solid lines). The polar angle $\theta$ represents the angle between the position vector and the first eigenvector of the local tidal field. In each panel the solid, dotted, dashed, long-dashed, dot-dashed, and dot-longdahsed line correspond to the range, $0^{\circ} \leq \theta<15^{\circ}$, $15^{\circ} \leq \theta<30^{\circ}, 30^{\circ} \leq \theta<45^{\circ}, 45^{\circ} \leq \theta<60^{\circ}, 60^{\circ} \leq \theta<75^{\circ}$ and $75^{\circ} \leq \theta \leq 90^{\circ}$, respectively. The anisotropic correlation functions of $\delta$ are also shown for comparison (thin solid lines). 


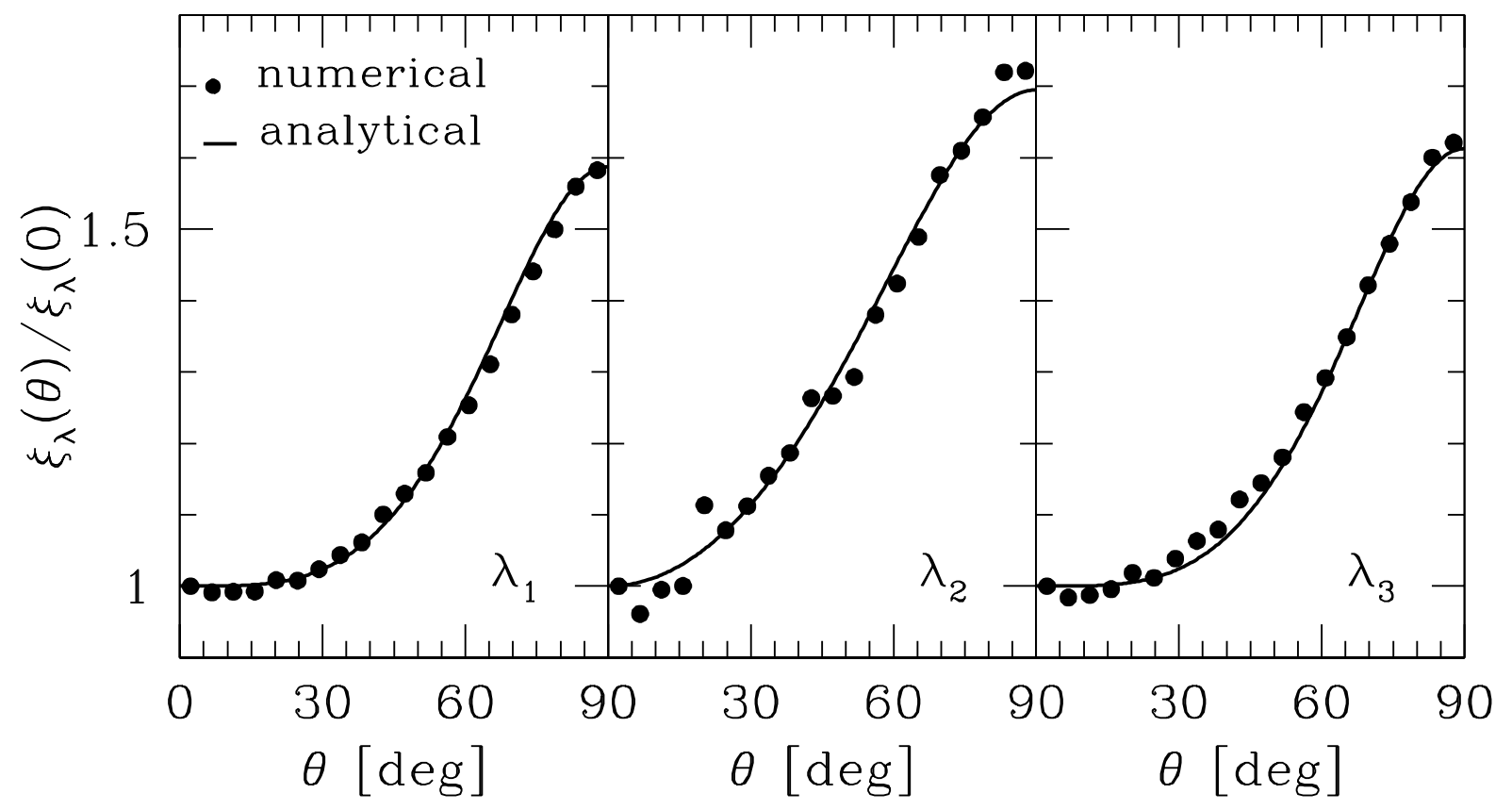

Fig. 5.- Anisotropic correlation function of $\lambda_{1}$ (right), $\lambda_{2}$ (middle) and $\lambda_{3}$ (left) as a function of $\theta$ in the principal axis frame of the local tidal field. In each panel the dots represent the numerical results while the solid line is the analytic fitting formula. 
Table 1. Best-fit values of the parameters in the fitting formula for the two-point anisotropic correlation functions of the three eigenvalues of the nonlinear traceless tidal field.

\begin{tabular}{cccc}
\hline \hline parameters & $\lambda_{1}$ & $\lambda_{2}$ & $\lambda_{3}$ \\
\hline$n$ & 1.28 & 2.76 & 1.65 \\
$r_{0}$ & 3.03 & 12.58 & 21.51 \\
$A$ & 0.37 & 0.41 & 0.38 \\
\hline
\end{tabular}

